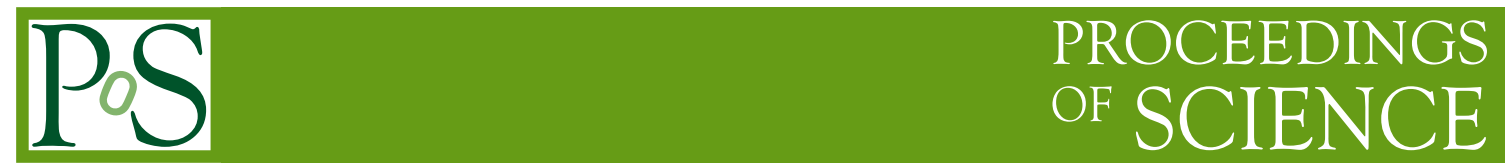

\title{
QED in three dimensions and at finite temperature
}

\author{
Eric Swanson* \\ University of Pittsburgh \\ E-mail: swansone@pitt.edu
}

\section{Pok Man Lo}

University of Pittsburgh

E-mail: pol4 @pitt.edu

\begin{abstract}
Novel nonperturbative properties of QED in three dimensions are examined. In accordance with earlier results, we establish that chiral symmetry is dynamically broken when the number of fermion species is sufficiently small. We also demonstrate that parity symmetry is broken in a certain region of parameter space. Subtleties of defining the theory at finite temperature are discussed.
\end{abstract}

XLIX International Winter Meeting on Nuclear Physics 24-28 January 2011

BORMIO, Italy

${ }^{*}$ Speaker. 


\section{QED3}

A variety of novel features has spurred interest in low-dimensional QED for many decades[1]. For example, high temperature QCD can be represented as the dimensionally reduced QCD3. If the number of quark flavours $\left(N_{f}\right)$ is large, the nonabelian behaviour of the theory is suppressed and it may be approximated as quantum electrodynamics in three dimensions (QED3)[2]. Massless QED3 in the large $N_{f}$ limit generates dynamical fermion masses that are suppressed exponentially in the fermion number. Thus this theory illustrates how large mass hierarchies can be dynamically generated[3], which is of interest to BSM physics.

More recently, QED3 has been used as a model field theory for three dimensional condensed matter systems. Examples include applications to high $T_{c}$ superconductors, where the relevant dynamics is thought to be isolated to copper-oxygen planes in the cuprate[4]. It is also considered as a gauge formulation of the $2+1$ dimensional Heisenberg spin model[5], a possible model for graphene[6], and quantum versions of spin-ice[7].

When coupled to $N_{f}$ massless four-component fermions, QED3 exhibits a $U\left(2 N_{f}\right)$ symmetry that can be broken to $U\left(N_{f}\right) \times U\left(N_{f}\right)$, which is the three dimensional analogue of chiral symmetry breaking. It is widely agreed that symmetry breaking occurs for low $N_{f}$. More interesting is the behaviour as the number of fermions becomes large where it is possible that the interaction becomes sufficiently screened that vacuum condensation no longer occurs. Indeed, Appelquist et al. have used the large $N_{f}$ limit with additional approximations to argue that there is a critical number of flavours, $N_{\star} \approx 3.5$ above which the theory remains in the symmetric phase. Furthermore, the lack of massless scalar bound states at the critical coupling has been used to argue that the chiral restoration phase transition is not second order, but is of a novel type[8].

The belief that a critical number of fermion flavours exists is not without controversy. For example, Pennington and Walsh assert that wavefunction renormalisation (which is null as $N_{f} \rightarrow \infty$ ) is central to determining the details of symmetry breaking. Their solution of a truncation of the Schwinger-Dyson equations reveals that, in fact, chiral symmetry is never restored[9]. In addition, Pisarski has used the renormalisation group to argue that fermion mass generation occurs for all values of $N_{f}[10]$. In spite of these claims, more recent Schwinger-Dyson computations again find a critical value of $N_{f}$ with $N_{\star} \approx 3.5[11,12]$.

A novel feature of QED3 is that it is possible to introduce a topological Chern-Simons-like photon mass term to the theory[13]. This term breaks parity and time reversal symmetries. It is also possible to formulate the theory with two-component fermions. In this case a nonzero photon mass induces a finite fermion mass at one-loop (and vice versa) $[14,15]$. This raises the interesting possibility that parity symmetry can be spontaneously broken in the massless theory. This question was first examined by Appelquist et al. many years ago[15]. They concluded that finite fermion masses were dynamically generated, but that these masses appear in pairs of opposite sign, thereby maintaining the parity symmetry of the vacuum and a massless photon, in agreement with a general argument of Vafa and Witten[16]. Their conclusions were based on an analytic examination of the Schwinger-Dyson equations in rainbow-ladder approximation. In addition the authors assumed the large $N_{f}$ limit, that there is no fermion wavefunction renormalisation, that the fermion self energy is constant at low momentum, and that it is valid to truncate integrals at the scale $e^{2}$. Their general conclusions were confirmed by other groups with similar methods[17]. A brief review of these 
issues and the extension to nonzero temperature are described in the following.

\section{Zero Temperature}

We study massless abelian gauge theory in three dimensions with two-component fermions[18]. The Lagrangian is taken to be

$$
\mathscr{L}=-\frac{1}{4} F^{2}+\bar{\psi}(i \partial d+e A) \psi-\frac{1}{2 \xi}(\partial \cdot A)^{2} .
$$

The coupling $e^{2}$ has units of mass and the theory is superrenormalisable. As mentioned in the introduction, a fermion mass term generates a topological photon mass

$$
\mathscr{L}_{\mathrm{CS}}=\mu \frac{1}{4} \varepsilon_{\mu v \alpha} F^{\mu v} A^{\alpha}
$$

via radiative corrections. Similarly, a nonzero value for $\mu$ will generate a fermion mass at one loop[13, 14].

The full photon propagator is given by the expression

$$
D_{\mu v}=\frac{-i(1-\Pi)}{p^{2}(1-\Pi)^{2}-(\mu-\tilde{\Pi})^{2}}\left(P_{\mu v}-i \frac{\mu-\tilde{\Pi}}{p^{2}(1-\Pi)} \varepsilon_{\mu v \alpha} p^{\alpha}\right)-i \xi \frac{p_{\mu} p v}{p^{4}}
$$

where the projector $P_{\mu \nu}$ is defined as

$$
P_{\mu v}=g_{\mu v}-\frac{p_{\mu} p_{v}}{p^{2}}
$$

and the full photon self energy is parameterised as

$$
\Pi_{\mu v}(p)=p^{2} P_{\mu v} \Pi(p)+i \varepsilon_{\mu v \alpha} p^{\alpha} \tilde{\Pi}(p) .
$$

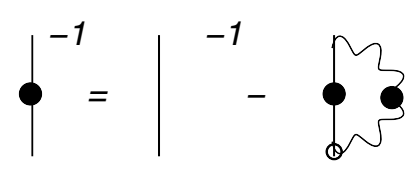

$$
\xi^{-1}=\xi^{-1}-\xi_{\xi}
$$

Figure 1: Schwinger-Dyson Equations. Solid circles represent full propagators. The open circles represent a model vertex.

The Schwinger-Dyson equations for the two-point functions are shown in Fig. 1. Solutions to these equations yield the full photon propagator, parameterised in Eq. 2.3 and the full fermion propagator, defined by

$$
S(p)=\frac{i}{A(p) p-B(p)} .
$$

The Schwinger-Dyson equations have been truncated by assuming a model form for the vertex (denoted with a small open circle). Typical model vertices include the rainbow ladder approximation:

$$
i \Gamma_{R L}^{\mu}(k, p)=\gamma^{\mu},
$$


the central Ball-Chiu vertex

$$
i \Gamma_{C B C}^{\mu}(k, p)=\frac{1}{2}(A(k)+A(p)) \gamma^{\mu}
$$

or the Ball-Chiu vertex

$$
i \Gamma_{B C}^{\mu}(k, p)=\frac{1}{2}(A(k)+A(p)) \gamma^{\mu}+\frac{1}{2} \frac{A(k)-A(p)}{k^{2}-p^{2}}(k+p)\left(k^{\mu}+p^{\mu}\right)-\frac{B(k)-B(p)}{k^{2}-p^{2}}\left(k^{\mu}+p^{\mu}\right) .
$$

The Ball-Chiu vertex is the unique form of the longitudinal portion of the vertex that is consistent with the Ward-Takahashi identity and is free of kinematic singularities[19]. Use of the Ball-Chiu vertex is a necessary, but not sufficient, condition for gauge invariance of the solution.

The transverse portion of the full vertex remains unspecified. Curtis and Pennington have used multiplicative renormalisability to argue that the most important transverse term is[20]

$$
i \Gamma_{C P}^{\mu}(k, p)=\frac{1}{2} \frac{A(k)-A(p)}{d(k, p)}\left[\gamma^{\mu}\left(k^{2}-p^{2}\right)-(k+p)^{\mu}(k-p)\right]
$$

with

$$
d(k, p)=\frac{\left(k^{2}-p^{2}\right)^{2}+\left(M(k)^{2}+M(p)^{2}\right)^{2}}{k^{2}+p^{2}}
$$

where $M=B / A$ is the mass term of the full propagator. We shall refer to these truncations as RL (rainbow-ladder), CBC (central Ball-Chiu), BC (Ball-Chiu), or CP (following Ref. [12], this is the Ball-Chiu plus Curtis-Pennington vertex in the fermion propagator and the Ball-Chiu vertex in the photon propagator).

\subsection{Chiral Symmetry Restoration}

We examine the putative phase transition more closely by plotting the fermion condensate versus $N_{f}$ (Fig. 2) for the RL, CBC, BC, and CP models. Also shown are fits with the Ansatz

$$
\langle\bar{\psi} \psi\rangle\left(N_{f}\right)=a N_{f} \exp \left(\frac{-2 \pi}{\sqrt{N_{\star} / N_{f}-1}}\right) .
$$

It is quite difficult to obtain these results for large values of $N_{f}$ : extrapolation in the number of integration points is necessary for $N_{f}>4$. Our most accurate results are for the CBC vertex and are in excellent agreement with the Ansatz of Eq. 2.12, implying strongly that a chiral restoration phase transition does indeed occur. An Ansatz like $\langle\bar{\psi} \psi\rangle \propto \exp \left(-N_{f}^{4}\right)$ also does a reasonable job fitting the CBC points, and of course does not exhibit a phase transition. Nevertheless the quality of this fit is significantly lower than that of Eq. 2.12.

Remarkably, a simple adjustment of $N_{\star}$ in Eq. 2.12 provides excellent fits to the RL, BC, and CP data as well. We obtain $a=1.8$ and

$$
\begin{gathered}
N_{\star}(\mathrm{CBC})=1.00 \cdot N_{\star}^{A} \\
N_{\star}(\mathrm{RL})=1.10 \cdot N_{\star}^{A} \\
N_{\star}(\mathrm{CP})=N_{\star}(\mathrm{BC})=1.21 \cdot N_{\star}^{A} .
\end{gathered}
$$




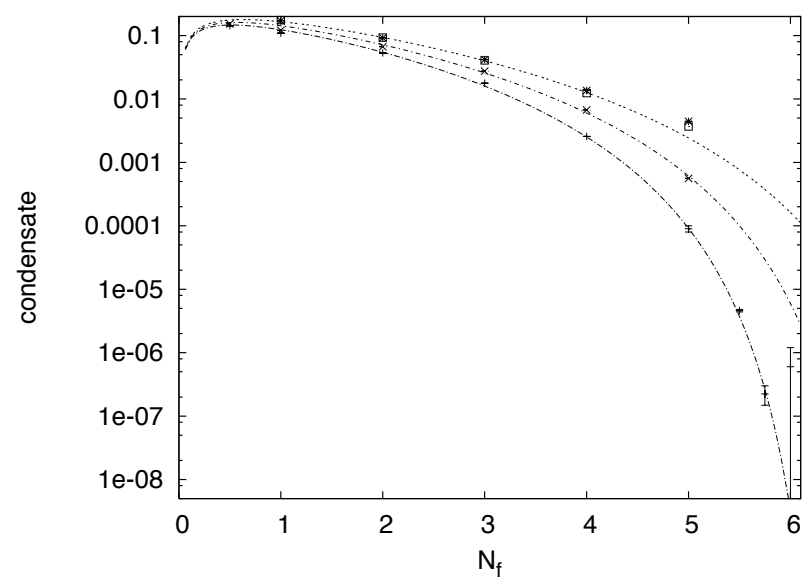

Figure 2: The condensate for (top to bottom) BC, CP, RL, and CBC models, with fit functions (described in the text).

Although we can be less certain that the BC and CP models exhibit symmetry restoration, the good fits, the similarity of all model results, and the theoretical motivation all make this very likely in our opinion. Our numerical results appear to be in agreement with earlier results [11, 12].

The seemingly good fit of the RL results (although we did not push the computation beyond $N_{f}=5.0$ ) illustrates the difficulty in extrapolating numerical results such as these. This is because it has been shown that the simple RL vertex Ansatz cannot support symmetry restoration[21].

\subsection{Parity}

We seek to confirm the reflection symmetry breaking pattern suggested by Appelquist et al.. Thus if parity symmetry is maintained one expects either no chiral symmetry breaking or chiral symmetry breaking with $N_{f} / 2$ fermions of mass $M$ and $N_{f} / 2$ fermions of mass $-M$. If reflection symmetry is maximally broken then one expects $N_{f}$ fermions with mass $M$ (or $-M$ ). One can also obtain parity breaking with intermediate scenarios.

We have performed computations assuming a reflection symmetry breaking pattern of $(M, M)$ with all vertex models[18]. The natural iteration algorithm approaches a limit cycle of the type $(A, B) \rightarrow(A,-B)$. While this could prove to be a nontrivial solution (due to the $B$ reflection symmetry discussed above), the error does not decrease with iteration. This suggests that the limit cycle does not represent an actual solution to the Schwinger-Dyson equations. Of course, the lack of convergence of a natural iteration algorithm does not prove that a solution does not exist. We have therefore repeated the computation with the minimisation algorithm. In this case $A$ evolves to a smooth function while $B$ approaches zero (with substantial noise). Similar behaviour was seen in all vertex models. Thus it appears that a nontrivial reflection-breaking solution to the Schwinger-Dyson equations does not exist, as suggested in Refs.[15], [17]. This computation is the first extension of those conclusions to the full two-point function Schwinger-Dyson equations with vertex models of varying sophistication.

We now seek to generalise these results to all patterns of symmetry breaking. Thus we introduce the parameter 


$$
\eta=\frac{N_{+}-N_{-}}{N_{+}+N_{-}}
$$

where $N_{ \pm}$is the number of fermions with mass $\pm M$. As discussed above, when $\eta=0$ and $M>0$ one obtains a parity-preserving, chiral symmetry-breaking vacuum structure. When $\eta=1$ the vacuum is maximally parity-violating. In general we find three types of behaviours: (i) iteratively stable solutions, (ii) limit cycles that are not solutions, (iii) chaotic results that are not solutions. Results for the rainbow-ladder and CBC vertices are shown in Fig. 3. In the rainbow-ladder case all results for $\eta>0.4$ are of the limit-cycle type (shown as two values for $B(0)$ in the figure). Surprisingly, stable parity-violating solutions exist for $\eta<0.4$. Similar behaviour is seen with the $\mathrm{CBC}$ vertex, except that the transition point is near $\eta=0.35$ and chaotic results are seen for greater values of $\eta$, with the exception of the maximally parity violating scenario, where a limit cycle behaviour is obtained. Results for the Ball-Chiu vertex are similar to those of the CBC vertex. The figure suggests that the transition between parity-preserving and parity-violating vacua occurs at $B(0)=0$, ie., when chiral symmetry is restored.

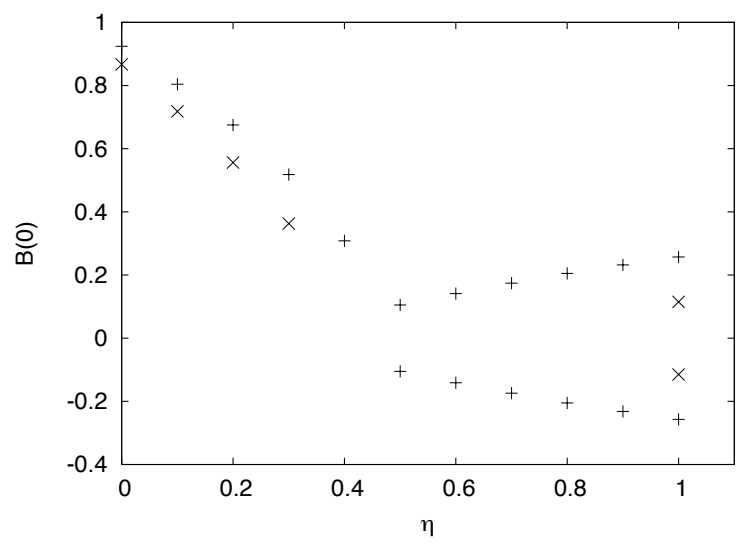

Figure 3: Parity breaking solutions vs. $\eta$. Plusses are RL, crosses are CBC.

\section{Finite Temperature}

The extension of a reliable computational scheme for QED3 to finite temperature is of interest due to its many condensed matter applications. This is a technically challenging problem, and past attempts have been forced to make many additional approximations beyond the truncation of the Schwinger-Dyson equations. The most immediate concern is the lack of covariance which makes dynamical quantities a function of two variables, $p_{0}, \vec{p}$, rather than simply $p^{2}$. This raises the computational requirements by one or two orders of magnitude.

An additional concern is the presence of infrared divergences in the formalism. Infrared divergences are exacerbated at nonzero temperature because perturbative diagrams are dominated in the infrared limit by the lowest available Matsubara frequency, which is zero in bosonic sums. Thus, even though QED3 is infrared finite at zero temperature, problems may arise again at nonzero temperature. This issue has engendered some confusion in the literature. Some authors have noted that an infrared divergence exists, but have ignored it[22], or imposed an infrared cutoff[23], or 
assumed that higher order corrections remove the divergence[24]. Many authors simply evade the issue entirely by employing the approximation[25]

$$
i D_{\mu \nu}(\omega, \vec{q}) \rightarrow i D_{00}(0, \vec{q}) .
$$

We shall argue that infrared divergences are endemic to QED3 at finite temperature. Furthermore, the problem is not alleviated by finite fermion masses. Nevertheless, observables are finite and the theory is well-defined.

We employ the imaginary time formalism and choose to work covariantly, which necessitates introducing a three-vector, $n^{\mu}$, that represents the heat bath. Thus the full fermion propagator is

$$
S=\frac{i}{\left(A-A_{0}\right) \not 1+A p-B} .
$$

Here

$$
p^{\mu}=\left(i \omega_{n}, \vec{p}\right)
$$

where $\omega_{n}=(2 n+1) \pi T$ is a fermionic Matsubara frequency and $A, B$, and $A_{0}$ are functions of $\omega_{n}$ and $\vec{p}$.

\subsection{Photon Propagator}

The vacuum polarisation tensor remains transverse at finite temperature, however the presence of an additional three-vector permits two transverse tensors

$$
P_{\mu v}^{L}(n, q)=\hat{q}_{\mu}^{\perp} \hat{q}_{v}^{\perp}
$$

and

$$
P_{\mu v}^{\perp}(n, q)=g_{\mu v}-\frac{q_{\mu} q_{v}}{q^{2}}-P_{\mu v}^{L}(q) .
$$

A transverse three-vector has been defined as

$$
q_{\mu}^{\perp}=q_{\mu}-n_{\mu} \frac{q^{2}}{n \cdot q} .
$$

Some useful properties of these tensors are

$$
\begin{array}{ccc}
q^{\mu} P_{\mu v}^{L}(q)=q^{\mu} P_{\mu v}^{\perp}(q)=0 & n^{\mu} P_{\mu \nu}^{\perp}=0 \\
P^{\perp} \cdot P^{L}=0 & P^{\perp} \cdot P^{\perp}=P^{\perp} & P^{L} \cdot P^{L}=P^{L} .
\end{array}
$$

With $n^{\mu}=(1,0,0)$ we obtain

$$
P_{00}^{\perp}=0 \quad P_{0 i}^{\perp}=0 \quad P_{i j}^{\perp}=-\left(\delta_{i j}-\hat{q}_{i} \hat{q}_{j}\right) .
$$

The minus sign in the last expression is required in the covariant normalisation employed here.

With these definitions one can parameterise the photon self energy as: 


$$
\begin{aligned}
\Pi_{\mu v}(n, q)= & P_{\mu v}^{\perp} \Pi_{\perp}+P_{\mu v}^{L} \Pi_{L}+i \varepsilon_{\mu v \alpha} \hat{q}^{\alpha} \tilde{\Pi}+i \hat{q}_{\mu}^{\perp} \varepsilon_{v \alpha \beta} \hat{q}^{\alpha} \hat{q}_{\perp}^{\beta} \Pi_{4}+i \hat{q}_{v}^{\perp} \varepsilon_{\mu \alpha \beta} \hat{q}^{\alpha} \hat{q}_{\perp}^{\beta} \Pi_{5}+ \\
& +\varepsilon_{\mu \alpha \beta} \varepsilon_{v \alpha^{\prime} \beta^{\prime}} \hat{q}^{\alpha} \hat{q}^{\alpha^{\prime}} \hat{q}_{\perp}^{\beta} \hat{q}_{\perp}^{\beta^{\prime}} \Pi_{6}
\end{aligned}
$$

Note that $\tilde{\Pi}, \Pi_{4}, \Pi_{5}$, and $\Pi_{6}$ are all null for four-component fermions and it is possible to combine the $\Pi_{4}$ and $\Pi_{5}$ terms into symmetric and antisymmetric tensors.

The full photon propagator is

$$
\begin{aligned}
i D_{\mu v}(n, q)= & D_{\perp} P_{\mu v}^{\perp}+D_{L} P_{\mu v}^{L}-i \xi \frac{q_{\mu} q_{v}}{q^{4}}+i \tilde{D}_{\mu v \alpha} \hat{q}^{\alpha}+i D_{4} \hat{q}_{\mu}^{\perp} \varepsilon_{v \alpha \beta} \hat{q}^{\alpha} \hat{q}_{\perp}^{\beta}+i D_{5} \hat{q}_{v}^{\perp} \varepsilon_{\mu \alpha \beta} \hat{q}^{\alpha} \hat{q}_{\perp}^{\beta}+ \\
& +D_{6} \varepsilon_{\mu \alpha \beta} \varepsilon_{v \alpha^{\prime} \beta^{\prime}} \hat{q}^{\alpha} \hat{q}^{\alpha^{\prime}} \hat{q}_{\perp}^{\beta} \hat{q}_{\perp}^{\beta^{\prime}}
\end{aligned}
$$

\subsection{Infrared Divergences}

It is known that $\Pi_{L}$ is nonzero at zero momentum: this provides electric screening in-medium. The one-loop expression for the Chern-Simons form factor is

$$
\tilde{\Pi}^{(\mathrm{mat})}(0, q \rightarrow 0)=\alpha q \tanh (m / 2 T) .
$$

This result should be compared to the zero temperature form factor

$$
\tilde{\Pi}(0)=\alpha q \frac{m}{|m|} .
$$

We remark that both of these results hold to all orders due to a theorem of Coleman and Hill[26].

An old argument due to Fradkin[27] establishes that $\Pi_{\perp}$ is zero at $(\omega, \vec{p})=(0,0)$. This statement can be extended to

$$
\Pi_{\perp}(0, q \rightarrow 0)=c_{\perp} q^{2}+O\left(q^{4}\right) .
$$

This is explicitly true to $O\left(e^{5}\right)$ in QED4 and Blaizot et al. argue that it is true to all orders[28]. The basic idea is that the nonvanishing minimum fermionic Matsubara frequency makes the self energy an analytic function of $q^{2}$. An expansion about $q=0$ then yields $\Pi_{\perp} \rightarrow 0+O\left(q^{2}\right)$, with the odd terms vanishing due to rotational invariance. We note that this argument generalises directly to three dimensions. This result is important because it implies that there is no dynamical screening in the magnetic sector.

The lack of magnetic screening leads directly to an infrared divergence in the fermion selfenergy, as we now demonstrate. Consider the exact expression for the fermion self energy that appears in the Schwinger-Dyson equation of Fig. 1.

$$
i \Sigma(p)=e^{2} T \sum_{n} \int \frac{d^{2} q}{(2 \pi)^{2}} \gamma_{v} S(q) \Gamma_{\mu}(p, q) D^{\mu v}(p-q) .
$$

We again employ the finite temperature version of the Ward identity to obtain the leading behaviour of the fermion self energy when $q=p-\eta$ : 


$$
\Gamma_{v}(p+\eta, p)=\frac{\partial S^{-1}(p)}{\partial p_{v}}+\eta^{\alpha} \frac{\Gamma_{v}}{\partial p_{\alpha}}+\ldots
$$

The leading infrared behaviour is obtained when $v$ (or $q_{0}$ ) is zero, we therefore set $v=0$ in the following. One obtains

$$
\begin{aligned}
\operatorname{div} \Sigma(p)= & -i e^{2} T\left[\gamma_{v} S(p) \frac{\partial S^{-1}}{\partial p_{\mu}}\right] \operatorname{div} \int_{\Lambda_{I R}} \frac{d^{2} \eta}{(2 \pi)^{2}} D_{\mu v}(\eta) \\
= & i e^{2} T\left[\gamma_{v} \frac{\partial S}{\partial p_{\mu}} S(p)^{-1}\right] \\
& \cdot \operatorname{div} \int_{\Lambda_{I R}} \frac{d^{2} \eta}{(2 \pi)^{2}}\left[n_{\mu} n_{v} D_{L}+\frac{n_{\mu} n_{v}-g_{\mu v}}{2}\left(-D_{\perp}+D_{6}-i \frac{\xi}{\eta^{2}}\right)\right]
\end{aligned}
$$

For two-component fermions only the gauge term is infrared divergent if the photon mass is nonzero. If it is zero one has

$$
\operatorname{div} \Sigma(p)=i e^{2} T\left[\gamma_{v} \frac{\partial S}{\partial p_{\mu}} S(p)^{-1}\right] \frac{n_{\mu} n_{v}-g_{\mu v}}{4 \pi}\left[-i \frac{1}{1-c_{\perp}}-i \xi\right] \log \Lambda_{I R}
$$

Similarly for four-component fermions with $\mu=0$

$$
\operatorname{div} \Sigma(p)=i e^{2} T\left[\gamma_{v} \frac{\partial S}{\partial p_{\mu}} S(p)^{-1}\right] \frac{n_{\mu} n_{v}-g_{\mu v}}{4 \pi}\left[-i \frac{1}{1-c_{\perp}}-i \frac{c_{6}}{\left(1-c_{\perp}\right)\left(c_{6}+c_{\perp}-1\right)}-i \xi\right] \log \Lambda_{I R} .
$$

We have assumed that $\Pi_{6}(0, q \rightarrow 0) \rightarrow c_{6} q^{2}$. If there is a screening mass in this form factor one can take the limit as $c_{6}$ goes to infinity in Eq. 3.20.

Thus a logarithmic infrared divergence appears in the fermion propagator. This statement is exact, only relying on the Ward identity, the existence of $1 / q^{2}$ terms in the exact photon propagator, and general properties of the photon form factors. It is clear that a finite fermion mass does not change this conclusion. However, a finite photon mass regulates the transverse part of the propagator, leaving only the divergence in the gauge term. These expressions make it clear that the infrared divergence does not affect observables. For example, it is possible to choose a gauge to eliminate the divergences entirely.

\subsection{The Electric Screening Mass}

The electric and magnetic screening masses are defined in terms of the longitudinal and transverse form factors of Eq. 3.10

$$
\begin{gathered}
m_{e l}^{2}=\lim _{p \rightarrow 0} \Pi_{L}(\omega=0, p) \\
m_{m a g}^{2}=\lim _{p \rightarrow 0} \Pi_{\perp}(\omega=0, p) .
\end{gathered}
$$

Of course, as stated above, the magnetic screening mass should be zero. The electric screening mass is an experimental observable and hence should be infrared and ultraviolet finite. Here we 
run into a problem: the naive application of Eq. 3.22 yields an infinite result for $m_{l l}$. The problem can be traced to a few linked causes:

(i) a superrenormalisable field theory can still contain divergences (although only a finite number of diagrams diverge),

(ii) the zero temperature photon self energy tensor is given in terms of a scalar function as $\Pi_{\mu v}=P_{\mu v} \Pi$, if the regulator and truncation scheme respect gauge invariance. If this is not the case one must write

$$
\Pi_{\mu \nu}=g_{\mu \nu} \Pi_{\infty}+P_{\mu \nu} \Pi .
$$

The new scalar function diverges. This function was neglected in previous analyses by simply projecting it away.

(iii) the projection trick no longer works at finite temperature. To obtain a finite screening mass one must renormalise properly; the final, finite, expression is

$$
m_{e l}^{2}=\lim _{p \rightarrow 0}\left[\Pi_{L}(0, p)+\Pi_{\infty}(0, p)-\Pi(p)-\Pi_{\infty}(p)\right]
$$

(In fact, the presence of the $g_{\mu \nu}$ term requires a photon mass term $\frac{1}{2} \mu A_{v} A^{v}$ in the Lagrangian and this expression is more conveniently expressed at the renormalisation point $\omega=\mu, \vec{p}=0$ for $T>0$ and $p^{2}=\mu^{2}$ for $T=0$.)

Unfortunately, this expression is very difficult to evaluate numerically. The divergence in the finite and zero temperature portions must cancel. A simple cutoff regulator will not do since it is implemented differently in three dimensions and $2+1$ dimensions. Numerically implementing dimensional regularisation is also not sufficient because this method can only deal with logarithmic divergences, and we have linear divergences. A third possibility is Pauli-Villars regularisation. This has the benefit of maintaining gauge invariance and separately regulates the zero and finite temperature portions of $m_{e l}^{2}$, which is desirable. Unfortunately, this doubles the computational effort as fermion functions $\left(A_{0}, A, B\right)$ must be obtained numerically for the Pauli-Villars fermion. Furthermore, one must extrapolate to large Pauli-Villars mass to obtain the cutoff independent result. This is very inconvenient because obtaining accurate results for large fermion mass is difficult. A possible way out of this numerical morass is to implement a judicious subtraction.

These options are currently under investigation and we hope to be able to report first-ever predictions of the truncated Schwinger-Dyson equations without additional approximations for finite temperature gauge theories in the near future.

\section{Conclusions}

QED3 is a rich quantum field theory. We have used the Schwinger-Dyson equations with a variety of model Ansätze to confirm that chiral symmetry is dynamically broken for a small number of fermion species. We have also observed for the first time the possibility of dynamical parity breaking. This needs to be investigated more fully; specifically, the preferred vacuum should be determined. Finally, we have established that QED3 contains infrared divergences at finite temperature that cannot be avoided, although these should not affect observables. Problems with computing the electric screening mass have been highlighted, along with potential solutions. 


\section{Acknowledgments}

This research was supported by the U.S. Department of Energy under contract DE-FG0200ER41135 and an Andrew W. Mellon Predoctoral Fellowship.

\section{References}

[1] J. S. Schwinger, Phys. Rev. 128, 2425 (1962).

[2] R.D. Pisarski, Phys. Rev. D 29, 2423, (1984).

[3] T. W. Appelquist, M. J. Bowick, D. Karabali and L. C. R. Wijewardhana, Phys. Rev. D 33, 3704 (1986).

[4] N. Dorey and N.E. Mavromatos, Nucl. Phys. B 386, 614 (1992); I.J.R. Aitchison and N.E. Mavromatos, Phys. Rev. D 53, 9321 (1996); P. Ghaemi and T. Senthil, Phys. Rev. B 73, 054415 (2006).

[5] R. Dillenschneider and J. Richert, Phys. Rev. B 74, 144404 (2006).

[6] V. P. Gusynin and S. G. Sharapov, Phys. Rev. Lett. 95, 146801 (2005).

[7] C.L. Henley, Ann. Rev. Cond. Mat. Phys. 1, 179 (2010).

[8] T. Appelquist, J. Terning and L. C. R. Wijewardhana, Phys. Rev. Lett. 75, 2081 (1995).

[9] M. R. Pennington and D. Walsh, Phys. Lett. B 253, 246 (1991).

[10] R.D. Pisarski, Phys. Rev. D44, 1866 (1991).

[11] P. Maris, Phys. Rev. D 54, 4049 (1996).

[12] C. S. Fischer, R. Alkofer, T. Dahm and P. Maris, Phys. Rev. D 70, 073007 (2004).

[13] J. Schonfeld, Nucl. Phys. B185, 157 (1981).

[14] S. Deser, R. Jackiw and S. Templeton, Annals Phys. 140, 372 (1982) [Erratum-ibid. 185, 406 (1988)] [Annals Phys. 281, 409 (2000)].

[15] T. Appelquist, M. J. Bowick, D. Karabali and L. C. R. Wijewardhana, Phys. Rev. D 33, 3774 (1986).

[16] C. Vafa and E. Witten, Phys. Rev. Lett. 53, 535 (1984).

[17] K. Stam, Phys. Rev. D 34, 2517 (1986); S. Rao and R. Yahalom, Phys. Rev. D 34, 1194 (1986).

[18] P. M. Lo, E. S. Swanson, Phys. Rev. D83, 065006 (2011). [arXiv:1010.1244 [hep-ph]].

[19] J.S. Ball and T.W. Chiu, Phys. Rev. D 22, 2542 (1980).

[20] D. C. Curtis and M. R. Pennington, Phys. Rev. D 42, 4165 (1990).

[21] A. Bashir, A. Raya, I. C. Cloet, C. D. Roberts, Phys. Rev. C78, 055201 (2008). [arXiv:0806.3305 [hep-ph]].

[22] D. J. Lee, Phys. Rev. D 58, 105012 (1998).

[23] G. Triantaphyllou, JHEP, 3, 20 (1999).

[24] D. Lee and G. Metikas, Int. J. Mod. Phys. A 14, 2921 (1999). 
[25] N. Dorey and N.E. Mavromatos, Phys. Lett. B 266, 163 (1991).

N. Dorey and N.E. Mavromatos, Nucl. Phys. B 386, 614 (1992);

I. J. R. Aitchison and M. Klein-Kreisler, Phys. Rev. D 50, 1068 (1994);

M. He, H.-T. Feng, W.-M. Sun, H.-S. Zong, Mod. Phys. Lett. A 22, 449, (2007);

J. F. Li, Y. Q. Zhou, H. T. Feng, W. M. Sun and H. S. Zong, Mod. Phys. Lett. A 25, 2645 (2010)

[26] S. R. Coleman and B. R. Hill, Phys. Lett. B 159, 184 (1985).

[27] E. Fradkin, Proc. Lebedev Phys. Inst., 29, 7 (1965).

[28] J.-P. Blaizot, E. Iancu, and R. Parwani, Phys. Rev. D 52, 2543 (1995). 